\section{$\mathbf{m} / \mathbf{s}$}

médecine/sciences $1998 ; 14: 139-41$

\title{
LE POINT SUR LE SIDA EN 1998
}

\section{Pierre Sonigo}

\section{RÉFÉRENCES}

1. Coffin JM. HIV population dynamics in vivo: implications for genetic variation, $\mathrm{pa}-$ thogenesis, and therapy. Science $1995 ; 267$ : 483-9.

2. Sonigo P. Viral evolution, virulence and AIDS pathogenesis. Cell Pharmacol AIDS Science 1996 ; 3 : 91-6.

3. Perelson AS, Essunger P, Cao Y, Vesanen M, Hurley A, Saksela K, Markowitz M, Ho DD. Decay characteristics of HIV-1-infected compartments during combination therapy. Nature 1997 ; 387 : 188-91.

4. Chun TW, Carruth L, Finzi D, Shen X, Di GJ, Taylor H, et al. Quantification of latent tissue reservoirs and total body viral load in HIV-1 infection. Nature 1997 ; 387 : 183-8.

5. Fauci AS. Host factors and the pathogenesis of HIV-induced disease. Nature 1996 ; $384: 529-34$.

\section{ADRESSE \\ P. Sonigo : Génétique des Virus, ICGM-Cnrs UPR415, Institut Cochin de génétique molé- culaire, 22, rue Méchain, 75014 Paris, France.}

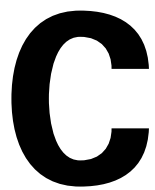

e numéro de médecine/ sciences fait une large place aux recherches consacrées au virus de l'immunodéficience humaine. En effet, notre connaissance des mécanismes qui contrôlent les équilibres hôtevirus au cours de l'infection par le VIH a largement progressé au cours des dernières années : on a pu, notamment, comprendre la signification des variations apparentes de la réplication virale et préciser les mécanismes d'entrée du virus dans la cellule. Ces éléments sont essentiels pour l'amélioration des thérapeutiques antivirales et portent l'espoir d'obtenir un jour la guérison du SIDA.

\section{Hôte et virus : redéfinir la latence}

Le virus est souvent considéré comme un être presque mort, parasite absolu et complexe dépendant de nos cellules pour assurer sa reproduction. Cette exploitation aboutirait, soit à une victoire virale et à la maladie, soit à une victoire de l'hôte et à l'absence de maladie. Ces deux situations caricaturales correspondent à des déséquilibres hôte-virus qui ne peuvent perdurer. En effet, le virus doit être considéré comme une forme de vie dépendant d'un écosystème cellulaire et de sa machinerie métabolique. Le système hôte-virus est stable lorsque le compromis optimal pour la survie de l'ensemble des partenaires est trouvé. Pris sous cet angle, rien ne distingue le virus, si ce n'est son extrême simplicité, d'un autre animal : par exemple d'un grand primate, parasite absolu d'un écosystème incluant la biochimie bactérienne et la photosynthèse végétale.
C'est dans ce contexte conceptuel que doit être abordée la notion de latence virale. Premièrement, il faut éviter de confondre la latence définie virologiquement, c'est-à-dire l'absence de réplication, avec la latence définie cliniquement, c'est-à-dire l'absence de symptomatologie: on sait maintenant que la période asymptomatique de l'infection VIH est le siège d'une intense activité réplicative (pour revue voir [1] et dans ce numéro de $\mathrm{m} / \mathrm{s})$. La latence réplicative est donc partielle car elle ne touche qu'une partie de la population virale. Elle est aussi dynamique car les virus latents à un instant ne sont pas ceux qui le seront à l'instant suivant, du fait de l'alternance latence-réplication au sein des populations de cellules infectées. Deuxièmement, il faut comprendre qu'un ralentissement de la multiplication virale permet d'augmenter la quantité de virus. Ce paradoxe tient au simple fait que la baisse de production virale diminue l'ardeur de la destruction immunologique : le nombre de virus survivants peut augmenter si la destruction baisse plus vite que la production. Par ce mécanisme, la réponse immune sélectionne les phénotypes viraux dits « lents » (pour revue voir [2]).

Du fait des conséquences en terme de stratégies thérapeutiques, la possible existence de virus latents, en arrêt réplicatif absolu pour de longues périodes de temps, constituant un sanctuaire inaccessible aux antiviraux « virustatiques » est un problème d'intense actualité. Sur le plan moléculaire, cela correspondrait à l'intégration du génome viral dans les chromosomes cellulaires et à l'arrêt de fonctionnement des gènes viraux. La capacité d'intégration est effectivement 
caractéristique des rétrovirus (voir l'article de R.E. Lodge $e t$ al., p. 148 de ce numéro). Restent à en déterminer l'importance quantitative et donc les conséquences au cours de l'infection VIH. En effet, on a pu estimer que les virus qui se répliquent représentent la grande majorité de la population infectante. Les cellules activement infectées ont une demi-vie de quelques jours et emportent les génomes intégrés dans leur tombe $\left(\mathrm{m} / \mathrm{s} n^{\circ} 6\right.$, vol. $12, p$. 820). Les cellules infectées de manière quiescente ont une demi-vie à peine plus longue (0,5 à 2 semaines [3]). Les génomes intégrés à demi-vie plus longue, facilement détectables par PCR sur l'ADN cellulaire, semblent incapables de se réveiller. La plupart ne sont que des déchets évolutifs résultant de mutations défavorables accumulées sur les gènes viraux: on parle de virus défectueux [4]. Un virus totalement latent à très long terme n'est donc qu'un virus « mort ».

Quelles que soient la précision de ces mesures et leurs conséquences sur la durée minimale des traitements, il faut réaliser que la latence n'est pas un phénomène de tout ou rien : entre un virus totalement "arrêté " et un autre "très réplicatif ", il existe probablement toute une gamme d'activités réplicatives et donc de sensibilités thérapeutiques. Les sanctuaires absolus définis dans une logique de tout-ou-rien ne peuvent correspondre à une réalité biologique : la taille du sanctuaire inaccessible et donc la durée minimale de traitement diminueront avec l'efficacité et l'agressivité de la thérapeutique antivirale.

\section{Un virus à charge variable}

La charge virale est une évaluation quantitative du nombre de virus présents dans l'organisme. Elle s'appuie sur la détection du matériel génétique viral (ARN génomique) qui offre une meilleure sensibilité que les tests d'antigénémie pratiqués auparavant, qui détectaient les protéines virales (p25gag). Ces tests ont constitué un énorme progrès dans le suivi de l'efficacité des thérapeutiques antivirales. En l'absence de traitement, l'évolution de la charge virale phases. Une phase précoce à charge virale élevée, qui correspond à la primo-infection, durant (en moyenne) 6 semaines. Une phase intermédiaire, à charge virale basse mais lentement progressive, de durée variable pouvant s'étaler sur de nombreuses années. Une phase terminale, correspondant au SIDA clinique, au cours de laquelle la charge virale s'élève à nouveau.

Les mécanismes de ces variations ont fait l'objet de nombreuses discussions dont l'objet n'est autre que la compréhension de la physiopathologie du déficit immunitaire (pour revue, voir [5]). Dans le passé, la baisse de charge survenant au décours de la primoinfection était attribuée à une entrée en latence virologique, sous le contrôle d'une " régulation » virale. L'enjeu était alors, pour éviter le passage à la phase clinique, d'élucider les co-facteurs "réglant " la remontée terminale de la charge. Comme trop souvent en biologie moléculaire, le mot « régulation » était invoqué abusivement pour habiller de science un profond vide conceptuel : l'entrée en latence puis, des années plus tard, le réveil synchrone de milliards de virus habitant des types cellulaires différents aurait relevé de la magie plus que de la régulation. Comme nous l'avons vu plus haut, la latence est aujourd'hui conçue sur un mode partiel, probabiliste et dynamique. Les variations de la charge ne font que refléter les variations de la sélection exercée par l'environnement immunologique du virus. Une immunité efficace élimine les virus, et sélectionne les plus lents d'entre eux : la charge est élevée et les virus sont rapides quand l'immunité n'est pas encore là (primo-infection), ou qu'elle a perdu son efficacité (phase tardive). Une charge virale basse traduit simplement l'efficacité de l'immunité antivirale (phase asymptomatique). La remontée de la charge virale n'est pas la cause, mais la conséquence du déficit immunitaire. Reste donc à expliquer l'origine de ce déficit.

On attribue aujourd'hui le SIDA à une atteinte progressive du tissu lymphoïde périphérique. Ce dernier fait l'objet d'une inflammation chronique due à la multiplication virale dans et autour des cellules de ce tis- su : macrophages, lymphocytes $\mathrm{CD} 4^{+}$, et cellules dendritiques (voir l'article de P. Champagne et al., p. 142 de ce numéro). Par analogie avec les hépatites chroniques avec cirrhose, on peut dire que la phase clinique terminale de l'infection par VIH traduit la décompensation des fonctions lymphoïdes. Le SIDA serait donc un déficit adéno-lymphatique plutôt qu'un déficit $\mathrm{T}$ pur lié à la baisse des lymphocytes CD4. Cette dernière ainsi que les autres désordres immunologiques observés au cours du SIDA (cytokines, activation cellulaire) seraient donc secondaires au déficit adéno-lymphatique (pour revue voir [5] et voir l'article de P. Champagne et al., p. 142 de ce numéro).

\section{Passer l'obstacle membranaire}

Le virus ne sort de la cellule qui l'a produit que pour entrer dans un nombre plus grand de cellules. La sortie et l'entrée des particules virales butent sur le franchissement des membranes cellulaires lipidiques. Ce franchissement est catalysé par les protéines d'enveloppe (pour revue voir [6]). Celles-ci sont elles-mêmes insérées dans une membrane lipidique qui délimite la particule virale (membrane virale). Virus et cellule peuvent donc être perçus comme deux «bulles » limitées par des lipides.

L'entrée correspond à une fusion de la « bulle » virale dans la « bulle » cellulaire. A l'inverse, la sortie est le bourgeonnement d'une petite bulle à partir d'une plus grosse. Un tel mécanisme d'entrée-sortie n'implique pas la lyse cellulaire dont de nombreux autres virus ont besoin pour sortir de la cellule. Pourtant le VIH détruit les cellules en culture. Cette destruction n'est pas une fin en soi : c'est un effet secondaire des propriétés de fusion membranaire de l'enveloppe. En effet, dans des conditions où l'enveloppe est abondante, telles que les systèmes de culture utilisés au laboratoire, cette protéine déclenche la fusion des membranes cellulaires entre elles : cela produit des syncytiums, perturbe les compartiments intracellulaires et aboutit à la mort de la cellule (effet cytopathogène).

Alors que le débat n'est pas tout à fait réglé pour savoir la part de cet effet 
dans la pathogénie, il apparaît que l'enveloppe est optimalisée en fonction de deux contraintes contradictoires : fusionner les membranes virales et cellulaires au moment de l'entrée du virus et ne pas les fusionner dans d'autres circonstances, notamment lors de la synthèse des protéines virales qui nécessitent des compartiments membranaires intacts (réticulum, Golgi).

Pour régler ce problème, les propriétés fusionnantes de l'enveloppe ne sont pas actives dès la synthèse de cette protéine. Par analogie, on peut considérer que la protéine initialement produite, appelée précurseur d'enveloppe ou gp160 (glycoprotéine de $160 \mathrm{kDa}$ ) est une pro-enzyme. Son activation ultérieure résulte de deux événements déclenchants: le premier est le clivage protéolytique qui se produit lors de son passage dans l'appareil de Golgi. Les protéases responsables sont cellulaires. Ce clivage produit deux protéines d'enveloppe : la sous-unité transmembranaire (TM ou gp41) qui reste ancrée dans la membrane virale et « retient » ainsi la sous-unité de surface (SU ou gp120) plus externe. Après ce clivage, l'enveloppe n'est toujours pas fusionnante. Elle doit subir une seconde étape d'activation. Cette seconde étape consiste à déformer l'ensemble (changement de conformation) pour dégager le «site actif " fusionnant (peptide de fusion), localisé sur la sous-unité transmembranaire. Notons que, alors que les séquences d'enveloppe sont extrêmement variables, ce "site actif fusionnant» est conservé dans des virus très différents du VIH (virus de la grippe) (pour une réference récente voir [7]).

Plusieurs mécanismes sont susceptibles de provoquer ce changement de conformation activateur: le plus connu est l'interaction de forte affinité de la protéine de surface (SUgp120) avec la molécule CD4 (pour revue voir [6]). L'interaction CD4-gp120 « dégoupille » la sous-unité transmembranaire et déclenche la fusion. Il est fort probable, même si cela n'est pas formellement démontré, que l'autre mécanisme activateur est l'acidification: pour la plupart des virus fusionnants, l'activation de l'enveloppe est déclenchée par l'acidification des phagolysosomes macrophagiques dans lesquels les virus se retrouvent rapidement. On distingue ainsi, en général, la fusion membranaire à la membrane externe que le VIH pratique à $\mathrm{pH}$ neutre en culture cellulaire, et la fusion à $\mathrm{pH}$ acide, au sein des phago-lysosomes, qui pourrait être exploitée par les virus à tropisme macrophagique et les isolats primaires du VIH.

L'identification d'une activité enzymatique fusionnante au sein de l'enveloppe transmembranaire a récemment ouvert de nouvelles perspectives thérapeutiques ou vaccinales anti-VIH : certains fragments de l'enveloppe transmembranaire ont montré d'efficaces propriétés antivirales ou vaccinales [7]. Concernant toujours les mécanismes d'entrée par fusion, il faut signaler la participation récemment révélée de protéines à sept domaines transmembranaires de la famille des récepteurs des chimiokines $\left(m / s n^{\circ} 2\right.$, vol. 13, $p$. 266) (pour revue, voir [8] et voir l'article de P. Champagne et al., p. 142 de ce numéro). On ne sait pas exactement comment ces protéines cellulaires activent l'entrée virale : est-ce en transmettant un signal d'activation cellulaire dont la nature et les conséquences restent à préciser ? ou est-ce tout simplement en modifiant la structure des lipides membranaires pour faciliter la catalyse fusionnante de l'enveloppe? Toujours est-il que les sujets porteurs d'une déletion dans le gène d'un de ces récepteurs aux chimiokines (CCR5) présentent une résistance relative à l'infection ou à la progression clinique (pour revue, voir [8] et voir l'article de P. Champagne et al., p. 142 de ce num»éro). Cela n'explique cependant qu'une partie des cas d'infection asymptomatique à long terme, dont le déterminisme reste inconnu.

\section{Guérir le SIDA}

Il y a encore peu de temps, la thérapeutique du SIDA était celle des infections opportunistes. Les trithérapies sont un énorme progrès mais l'objectif affiché aujourd'hui n'est pas de gérer au mieux des multithérapies à long terme, mais d'obtenir la guérison de l'infection VIH. Personne ne sait aujourd'hui si cet objectif est réalisable, et c'est bien pour cela que les recherches virologiques et cliniques doivent s'y attacher en priorité [9].

Finalement, la logique mise en œuvre pour l'optimalisation de combinaisons médicamenteuses dont l'efficacité est suivie sur des critères de charge virale pourrait aussi débloquer les approches de la vaccination anti-VIH. Jusqu'à présent, il était très difficile de comparer l'efficacité des préparations vaccinales testées chez l'animal, le critère d'efficacité retenu étant celui de la protection en toutou-rien. Au contraire, les mesures de charge virale pratiquées lors des infections d'épreuve animales permettent de quantifier le niveau de protection conféré par la vaccination et cela, même lorsque la protection est incomplète. L'AZT n'a pas été éliminée de la pharmacopée anti-VIH sous prétexte que son utilisation isolée a un impact partiel. De la même manière, des préparations vaccinales autrefois écartées car insuffisamment efficaces utilisées isolément pourraient servir de base à la mise au point de combinaisons vaccinales à efficacité accrue. Dans le domaine de la vaccination, il est indispensable de ne pas faire de faux pas, ni en avant par des essais cliniques prématurés, ni en arrière par un pessimisme abusif. Le vaccin reste la cé de l'éradication du SIDA dans le monde

\section{RÉFÉRENCES}

6. Freed EO, Martin MA. The role of HIV-1 envelope glycoproteins in virus infection. $J$ Biol Chem 1995 ; 270 : 23883-6.

7. Chan DC, Fass D, Berger JM, Kim PS. Core structure of gp41 from the HIV envelope glycoprotein. Cell 1997; $89: 263-73$.

8. Moore JP, Trkola A, Drajic T. Coreceptors for HIV-1 entry. Curr Opin Ummunol 1997 ; 9 : 551-62.

9. Clavel F. Nouvelles données sur la dynamique de réplication du VIH in vivo: peuton envisager une éradication de l'infection par les traitements antiviraux? Med Sci 1997 ; $13: 1225-7$.

\section{TIRÉS À PART}

P. Sonigo. 